Gut, $1987,28,468-473$

\title{
Improved specificity of the PABA test with p-aminosalicylic acid (PAS)
}

\author{
F J HOEK, F A J T M VAN DEN BERGH, J T KLEIN ELHORST, \\ J L MEIJER, E TIMMER, AND G N J TYTGAT
}

From the Department of Gastroenterology, Academic Medical Centre, University of Amsterdam, Amsterdam, The Netherlands, and Clinical Chemistry Laboratory and Department of Internal Medicine, Hospital 'De Stadsmaten', Enschede, The Netherlands

Summary Until now use of the PABA test together with $\left[{ }^{14} \mathrm{C}\right] \mathrm{PABA}$ to calculate the PABA excretion index has probably been the best adaptation suggested to enhance the specificity of this non-invasive pancreatic function test. Drawbacks of the method are the application of radioactivity, the fact that children, pregnant women, and patients with renal insufficiency have to be excluded from the test, and the possible interference of drugs and isotopes. We propose simultaneous administration of p-aminosalicylic acid (PAS) in the PABA test and quantification of the urinary PABA and PAS excretion with liquid chromatography. Urinary PABA and PAS excretion in six hours are comparable $(69 \cdot 5 \pm 8 \cdot 4 \%$ and $65 \cdot 6 \pm 18 \cdot 4 \%$ respectively in five healthy volunteers). Application of the PABA/PAS ratio was compared with the urinary PABA excretion in 21 normal controls, 38 patients with pancreatic disease, and 42 patients without pancreatic pathology. The PABA/PAS ratio and the per cent PABA excretion correlated very well in pancreatic patients: (PABA/PAS ratio) $=0.0149(\%$ PABA $)+0.052(r=0.902)$. Use of the PABA/PAS ratio enhanced the specificity of the test from 76 to $89 \%$.

Since its introduction, the PABA test has gained an established position in the battery of tests available for the diagnosis of exocrine pancreatic insufficiency.' Advantages of the test are the fact that no invasive procedures are necessary and that simple colorimetric procedures are available for the quantification of p-aminobenzoic acid (PABA) in urine..$^{2-1}$.

It shares, however, a lack of specificity with other non-invasive and indirect pancreatic function tests. ${ }^{1}$ Falsely low excretion rates can be obtained by defects in gastric emptying, in endogenous stimulation of the pancreas, failure in raising the intestinal $\mathrm{pH}$, abnormalities in intestinal PABA resorption, in liver function and in renal excretion.

For a screening test to be used in a patient

Address for correspondence: Dr F J Hoek, Department of Gastroenterology (C2), Academic Medical Centre, Meibergdreef 9, 1105 AZ Amsterdam, The Netherlands.

Received for publication 28 July 1986. population with a low prevalence of the disease, a high specificity is of the utmost importance to obtain an acceptable predictive value of a positive result. Administration of an equimolar dose of free PABA on a separate occasion and calculation of a PABA excretion index has been recommended to compensate for abnormalities in PABA resorption and metabolism. ${ }^{5}$ With $\left[{ }^{14} \mathrm{C}\right]$ PABA the duration of the procedure can again be brought back to six hours, ${ }^{67}$ but the radioactivity involved excludes use of the test in children, pregnant women, and patients with renal insufficiency and prevents its widespread use. No single report is available in the literature of use of this test modification outside the UK. Besides, drug or isotopic interference inhibits test interpretation in about $10 \%$ of the cases. ${ }^{\text {}}$

Determination of PABA with high performance liquid chromatography (HPLC) is a simple and accurate procedure ${ }^{910}$ with the advantage of high analytical specificity. It permits the incorporation 
into the PABA test of a PABA-like marker substance. Calculation of the ratio of PABA and marker excretion should then eliminate influences of resorption, metabolism and excretion of PABA on the PABA test in the same way as the PABA excretion index does. A one day tubeless pancreatic function test is then obtained without radioactivity.

We propose p-aminosalicylic acid (PAS) as the marker substance, because it has a molecular structure very similar to PABA, consequently similar pharmacokinetic properties and it is readily separated from PABA in our HPLC assay. ${ }^{10}$ In this study data are presented of the PABA/PAS ratio, obtained in healthy volunteers and patients and a comparison is made with the results of the PABA test, expressed in the usual way.

\section{Methods}

\section{PATIENTS}

The PABA test was performed with $1 \mathrm{~g} \mathrm{~N}$-benzoyl-Ltyrosyl-PABA (BTPABA; bentiromide; HoffmanLa Roche, Basle Switzerland). The substrate and 360 $\mathrm{mg}$ PAS ( $500 \mathrm{mg}$ PAS Na. $2 \mathrm{H}_{2} \mathrm{O}$ ) were administered together with a Lundh test meal $(150 \mathrm{ml})$. Urine was collected during six hours. In approximately one half of the tests the urine was collected in separate one hour portions. In the other patients and controls the urine was collected in one 6 hour portion. Always a urine sample from before the start of the test was analysed also to ensure the absence of substances which could interfere with the analysis.

After alkaline hydrolysis of the PABA and PAS metabolites in the urine, analysis was undertaken by high performance liquid chromatography (HPLC) on a reversed phase column $(10 \mu \mathrm{m}$ Lichrosorb RP 18$)$ with a $3 / 1$ mixture (by vol) of $0.01 \mathrm{M} \mathrm{Na}$-acetate buffer $\mathrm{pH} 4.0$ and methanol as the mobile phase. Details of the procesure are described elsewhere. ${ }^{10}$

The amount of PABA recovered in the urine in six hours was expressed as a percentage of the PABA equivalents $(340 \mathrm{mg})$ administered orally as BTPABA. The lower limit of normal PABA excretion was $50 \%$. "The amount of PAS, which was recovered in the urine, was also expressed as a percentage of the $360 \mathrm{mg}$ PAS administered orally. The PABA/PAS ratio, the ratio of these percentages of recovered PABA and PAS was calculated.

The 12 healthy volunteers, participating in this study, were members of the hospital staff. Patient controls $(n=9)$ were without organic disease which could possibly interfere with the PABA test. For the pancreatic disease group, 38 patients were analysed with established disease. Pancreatic disease was confirmed by ultrasound, computed tomography, ERCP, endosonography, abdominal radiograph or histology. Diagnosis was chronic pancreatitis in 29 patients, pancreatic carcinoma in five patients, acute pancreatitis in three patients, and in one patient cystic fibrosis with pancreatic insufficiency. Pancreatic insufficiency was not always present. Faecal fat excretion was assessed in 21 patients and was abnormal in 18 . The majority of the 42 patients in the group with gastrointestinal disease was investigated because of upper abdominal complaints and/or diarrhoea. In these patients no independent proof of pancreatic involvement was obtained. In addition some patients with established malabsorption syndrome $(n=7)$, impaired gastric motility $(n=3)$, or severe liver cirrhosis $(n=3)$ were investigated.

\section{Results}

For use of PAS instead of PABA as internal reference of resorption, metabolism, and excretion, a comparison of their respective pharmacokinetics is essential. Therefore $340 \mathrm{mg}$ PABA and $360 \mathrm{mg}$ PAS ( $500 \mathrm{mg}$ PAS Na. $2 \mathrm{H}_{2} \mathrm{O}$ ) were administered together to five healthy volunteers with $150 \mathrm{ml}$ Lundh test meal. Urine was collected in one hour portions and PABA and PAS measured therein. Urinary excretion in six hours amounted to $69 \cdot 5 \pm 8 \cdot 4 \%(\bar{x} \pm 2$ SD) for PABA and to $65 \cdot 6 \pm 18 \cdot 4 \%(\bar{x} \pm 2$ SD) for PAS. Over the six hour period PABA and PAS excretion proved to be very comparable (Fig. 1). The PABA excretion was not different from data obtained in earlier experiments ${ }^{11}$ in five healthy volunteers after administration of $340 \mathrm{mg}$ PABA alone with $150 \mathrm{ml}$ Lundh test meal, measured with the colorimetric method. A different urinary excretion curve was

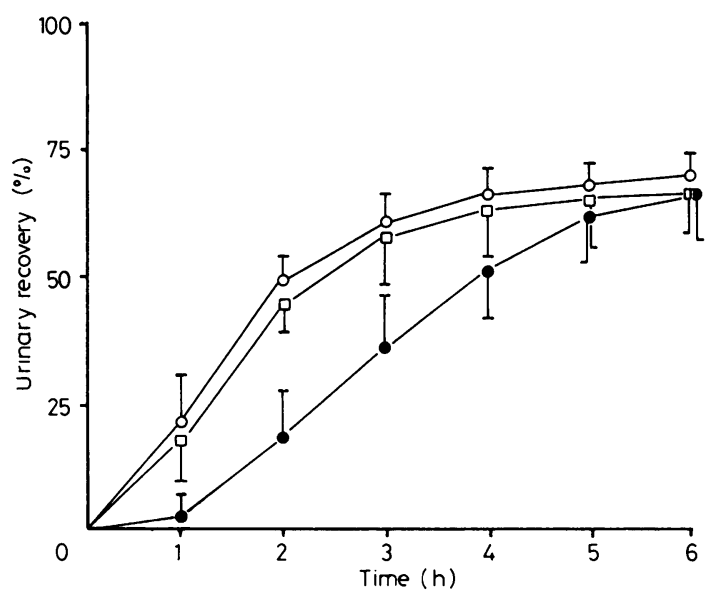

Fig. 1 Comparison of the time curves of urinary excretion of $P A B A$ and $P A S$. Mean $\pm S D$ are given after oral administration of $340 \mathrm{mg} P A B A(\mathrm{O}), 500 \mathrm{mg} P A S$ $\mathrm{Na} .2 \mathrm{H}_{2} \mathrm{O}(\square)$ and $1 \mathrm{~g} B T P A B A(\bigcirc)$ together with a test meal to 5 healthy volunteers. 
found for PABA, when it was administered as BTPABA with the test meal (Fig. 1), although the total excretion in six hours was equal.

The PABA/PAS ratio was evaluated in 12 healthy volunteers and nine patient controls, in 38 patients with evidence of disease of the exocrine pancreas and in 42 patients with gastrointestinal or liver disease without evidence of exocrine pancreatic involvement. After administration of $1 \mathrm{~g}$ BTPABA and $360 \mathrm{mg}$ PAS together with the test meal, urinary PABA excretion in the 12 healthy volunteers was $65 \cdot 3 \pm 20 \cdot 0 \%(\bar{x} \pm 2 \mathrm{SD})$. With the exception of one abnormal test result $(43.4 \%)$, this PABA excretion was in good agreement with the value reported earlier for 20 healthy volunteers, $74.0 \pm 18.8 \%$ $(\bar{x} \pm 2 \mathrm{SD})$ measured with the colorimetric method." PAS excretion in these healthy volunteers amounted to $74 \cdot 2 \pm 18 \cdot 3 \%(\bar{x} \pm 2 \mathrm{SD})$ of the administered dose. The PABA/PAS ratio for the 12 healthy volunteers was $0.89 \pm 0 \cdot 28(\bar{x} \pm 2 \mathrm{SD})$ with values ranging from 0.62 to $1 \cdot 15$.

In five healthy volunteers PAS excretion was measured once together with BTPABA and once with free PABA. Excretions measured were 74.7 and $73.3 \%, 65.7$ and $52.7 \%, 54.5$ and $59.0 \%, 79.7$ and $73.0 \%, 70 \cdot 1$ and $69 \cdot 8 \%$ respectively. Calculation of the PABA excretion index was also possible for these persons. Results for the PABA excretion index and the PABA/PAS ratio for these five volunteers are given in the Table.

Based on the results in the 21 normal controls 0.75 was arbitrarily accepted as lower limit of normal for the PABA/PAS ratio. The lower limit of normal for PABA excretion was $50 \%$. The results for the normal controls and the patients are given in Figure 2, expressed as per cent PABA recovered and as PABA/PAS ratio. In 25 of the 38 patients with pancreatic disease the PABA/PAS ratio was abnormal, giving a sensitivity of $66 \%$, while PABA excretion was $<50 \%$ in 28 patients $(74 \%)$. In the group of patients without pancreatic disease and the controls the PABA/PAS ratio was abnormal in seven of 63 cases, resulting in a specificity of $89 \%$, compared with an abnormal PABA excretion in 15 of the 63 cases (specificity $76 \%$ ). Results of the PABA/PAS

Table Comparison of $P A B A / P A S$ ratio and $P A B A$ excretion index $(P E I)$ in five healthy volunteers

\begin{tabular}{ll}
\hline PABA/PAS ratio & PEI \\
\hline 1.09 & 0.87 \\
0.91 & 0.93 \\
0.62 & 0.62 \\
0.91 & 0.99 \\
0.84 & 0.88 \\
\hline
\end{tabular}

ratio and the six hour PABA excretion are compared in Figure 3.

An excellent correlation between PABA/PAS ratio and $\mathrm{PABA}$ excretion was found for the patients with pancreatic disease: $($ PABA/PAS ratio) $=0 \cdot 0149$ $(\%$ PABA $)+0 \cdot 052(r=0 \cdot 902 ; n=38)$. The correlation was much weaker for the patients without pancreatic disease: $(\mathrm{PABA} / \mathrm{PAS}$ ratio $)=0.0056(\% \mathrm{PABA})+$ $0.556(r=0.510 ; n=42)$.

When a prevalence of pancreatic disease of $15 \%$ was assumed, ${ }^{\circ}$ a positive predictive rate was calculated of $35 \%$ for the PABA test and of $50 \%$ for the PABA/PAS ratio. The predictive value of a negative result for absence of pancreatic disease was $94 \%$ for both tests.

\section{Discussion}

It is well known from the literature that the limited specificity of the PABA test for exocrine pancreatic insufficiency can be improved by use of a PABA excretion index, to compensate for the influence of non-pancreatic factors on PABA excretion. Originally, this compensation was achieved by administration of an equimolar dose of free PABA on a different day and determination of its excretion as a reference. ${ }^{5}$ Later the use of a small dose of $\left[{ }^{14} \mathrm{C}\right]$ PABA was described. ${ }^{a-}$ This limited the procedure to six hours and eliminated the day to day variations in PABA excretion observed by Kay et $a l^{12}$ and ascribed by them to variations in gastric emptying. Radioactive compounds are, however, used only reluctantly and they prohibit use of the test in children, pregnant women, and patients with renal insufficiency.

The excretion index described by us here also has the advantage that it takes only a single six hour urine collection, but in addition it puts no restrictions on the patients to be investigated. For a reliable calculation of a PABA excretion index it is essential that the pharmacokinetic properties of the marker resemble the kinetics of PABA metabolism and excretion as closely as possible. Therefore we started to look for compounds with a molecular structure strongly related to PABA. PAS was chosen for the following reasons: (1) PAS differs from PABA only by one hydroxy-group. (2) In the literature data were available to prove its comparable metabolism ${ }^{13}$ and its high urinary excretion rate, ${ }^{14}$ supported later by our data reported here (Fig. 1). (3) PAS has been in use as a tuberculostatic drug for a long time in the recent past and much higher doses than used in this study have been administered safely to patients. Therefore we could be sure that no side effects were to be expected with the dose used in this study. This obviated the necessity of prior toxicologic studies. (4) 


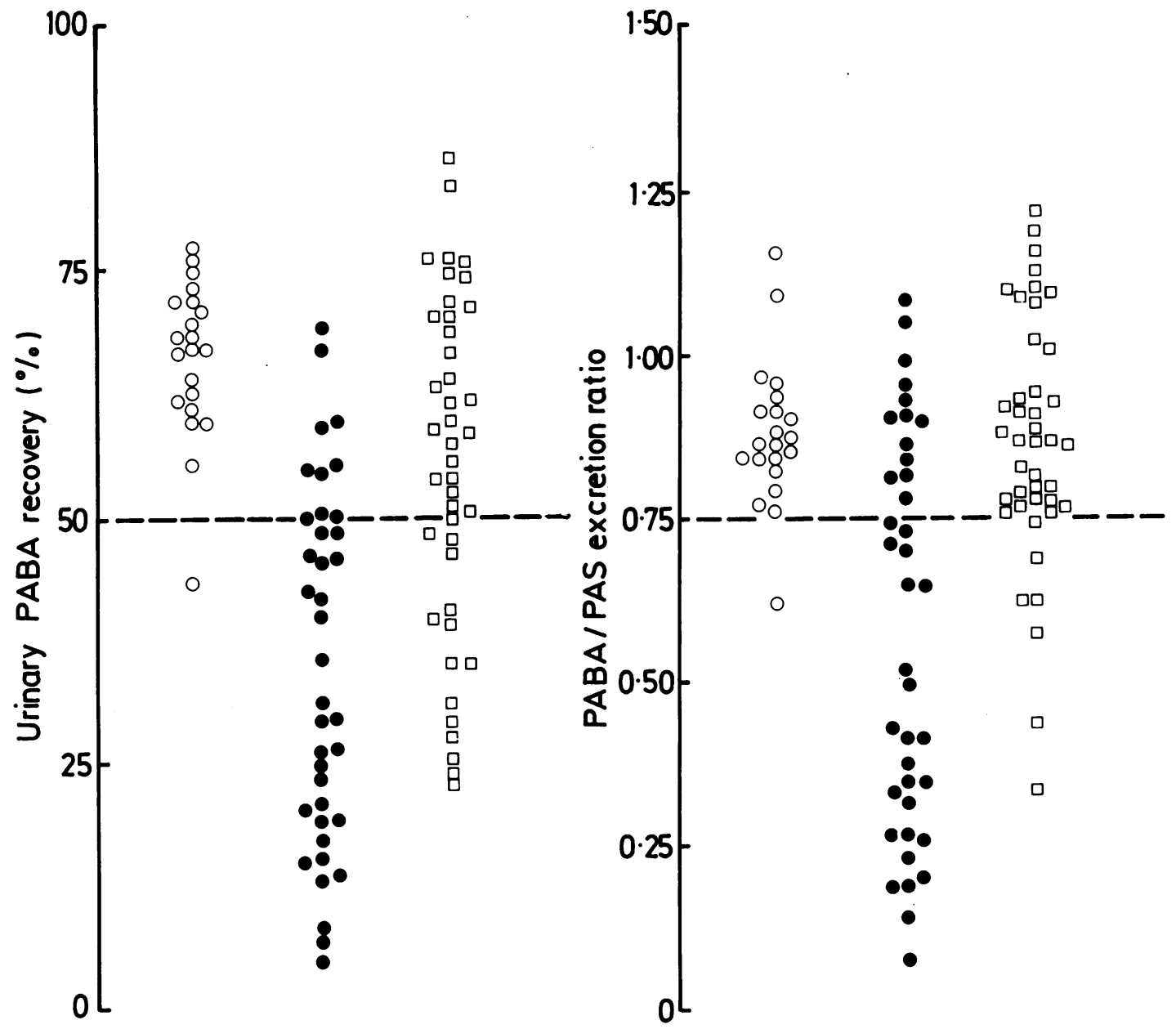

Fig. 2 Urinary PABA excretion and PABA/PAS ratio for normal controls $(\bigcirc)$, patients with pancreatic disease $(\Theta)$ and patients with gastrointestinal or liver disease $(\square)$. The dotted lines indicate the lower limits of the normal range.

In our HPLC assay for PABA, "' PAS was detected also with a retention time suitable for accurate quantification of both compounds.

As reported here for five healthy volunteers day to day variations in PAS excretion were not always negligible, confirming the day to day variations in PABA excretion reported by Kay et al. ${ }^{12}$ As a consequence, in one case a difference of 0.22 was found between PABA/PAS ratio and PABA excretion index (Table), although in the other case both parameters corresponded very well.

It may be pointed out here that small differences in excretion rate between the chosen marker and released PABA will not decrease the value of the excretion index. Also free PABA resorption and excretion are not completely parallel to the resorption and excretion of PABA, that has to be split off first from BTPABA in the duodenum. In healthy controls it was shown that with free PABA there was a smaller lag between oral ingestion and urinary excretion, even when taken with a meal" (Fig. 1). The difference may probably be explained by differences in gastric emptying of on the one hand free PABA, which is readily soluble at the low $\mathrm{pH}$ of the gastric contents, and on the other hand BTPABA, which is virtually insoluble at $\mathrm{pH}<5$. For $\mathrm{PAS}$, also acid soluble, only a slightly lower excretion rate was observed than for free PABA (Fig. 1) and PAS excretion differed therefore to the same extent as free PABA did from the excretion of PABA produced by BTPABA proteolysis in vivo.

The optimal dose of BTPABA is probably determined by the limited capacity of the body for handling PABA. ${ }^{1516}$ This limited PABA excretion 


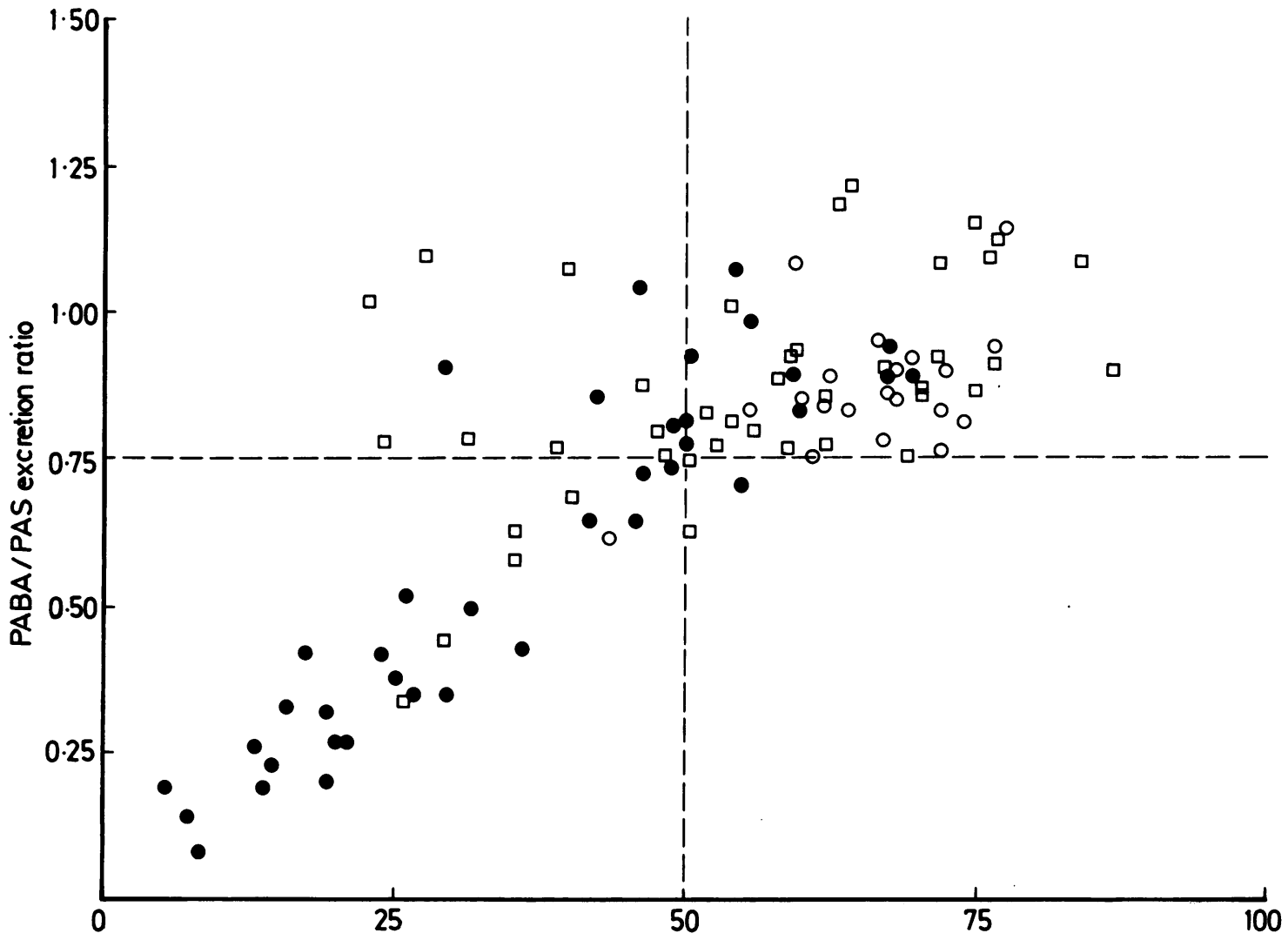

Fig. 3 Relationship between $P A B A / P A$ S excretion ratio and urinary $P A B A$ recovery. Results for 21 normal controls $(O), 38$ patients with pancreatic disease $(\Theta)$ and 42 patients with gastrointestinal or liver disease $(\square)$. The regression line for patients with pancreatic disease is: $(P A B A / P A S)=0.0149(\% P A B A)+0.052 ; r=0.902$. The dotted lines indicate the lower limits of the normal range.

capability has been suggested as an explanation for some false positive tests observed when a $2 \mathrm{~g}$ BTPABA dose was used. ${ }^{15}$ The urinary excretion curves for high doses of PAS reported in the literature $^{14}$ do not suggest a comparably limited capacity for PAS excretion. In the results obtained by us with combined administration of PAS and PABA, or PAS and BTPABA, no indications could be found for restricted PABA excretion. Still, the analytical precision of the HPLC procedure ${ }^{10}$ is such, that BTPABA and PAS doses may probably be halved without damage to the diagnostic accuracy of the test.

The data obtained in the patients and controls (Figs 2 and 3) support our opinion that PAS can be used instead of $\left[{ }^{14} \mathrm{C}\right] \mathrm{PABA}$ to obtain a reliable PABA excretion index. In almost all patients with pancreatic disease results for PABA excretion and PABA/PAS ratio corresponded very well. The specificity of the PABA test was raised by $13 \%$ by calculation of a PABA/PAS ratio, as expected from similar results reported with the PABA $/\left[{ }^{14} \mathrm{C}\right] \mathrm{PABA}$ excretion index. ${ }^{8}$ These encouraging results will have to be confirmed in more extensive patient studies.

\section{References}

1 Lankisch PG. Exocrine pancreatic function tests. Gut 1982; 23: 777-98.

2 Bratton AC, Marshall EK. A new coupling component for sulfonamide determination. J Biol Chem 1939; 128: 537-50.

3 Yamato Ch, Kinoshita K. A simple assay for measurement of p-aminobenzoic acid (PABA) in urine. In: Masuda M, ed. Pancreatic function diagnostant. Tokyo and New York: Igaku-Shoin, 1980: 138-42.

4 Eisenwiener HG, Morger F, Lergier W, Gillessen D. Die Bestimmung der p-Aminobenzoesäure mit Fluram ${ }^{\circledR}$ im Urin nach Durchführung des Pankreasfunktionstests mit. Bentiromid. J Clin Chem Clin Biochem 1982; 20: 557-65.

5 Mitchell CJ, Humphrey CS, Bullen AW, Kelleher J, Losowsky MS. Improved diagnostic accuracy of a 
modified oral pancreatic function test. Scand J Gastroenterol 1979; 14: 737-41.

6 Mitchell CJ, Field HP, Simpson FG, Parkin A, Kelleher J, Losowsky MS. Preliminary evaluation of a single-day tubeless test of pancreatic function. $\mathrm{Br}$ Med J 1981; 282: 1751-3.

7 Tetlow VA, Lobley RW, Herman K, Braganza JM. $A$ one day oral pancreatic function test using a chymotrypsin-labile peptide and a radioactive marker. Clin Trials J 1980; 17: 121-9.

8 Braganza JM, Kay GH, Tetlow VA, Herman KJ. Observations on the BTPABA $/{ }^{14} \mathrm{C}-\mathrm{PABA}$ tuneless test of pancreatic function. Clin Chim Acta 1983; 130: 339-47.

9 Ito S, Maruta K, Imai Y, et al. Urinary p-aminobenzoic acid determined in the pancreatic function test by liquid chromatography, with electrochemical detection. Clin Chem 1982; 28: 323-6.

10 Bergh FAJTM van den, Klein Elhorst JT, Hoek FJ, Tytgat GNJ. Rapid determination of the p- aminobenzoic acid excretion index in urine without use of radioactivity. Clin Chem 1986; 32: 234-5.

11 Hoek FJ, Sanders GTB, Teunen A, Tytgat GNJ. In vitro and in vivo analysis of the PABA test compared with the Lundh test-influence of intraluminal $\mathrm{pH}$. Gut 1981; 22: 8-14.

12 Kay GH, Tetlow VA, Braganza JM. Relationship between PABA and Lundh tests: lack of influence of duodenal pH in vivo. Clin Chim Acta 1983; 128: 115-24.

13 Way EL, Peng C-T, Allawala N, Daniels TC. The metabolism of p-aminosalicylic acid (PAS) in man. J Am Pharm Assoc 1955; :44: 65-9.

14 Wan SH, Pentikainen PJ, Azarnoff DL. Bioavailability of amino-salicylic acid and its various salts in humans III: absorption from tablets. J Pharm Sci 1974; 63: 708-11.

15 Imondi AR. Optimum dose of bentiromide. Dig Dis Sci 1983; 28: 859-60.

16 Drucker MM, Blondheim SH, Wislicki L. Factors affecting acetylation in vivo of para-aminobenzoic acid by human subjects. Clin Sci 1964; 27: 133-41. 